\title{
Uso Crônico de Decanoato de Nandrolona Como Fator de Risco Para Hipertensão Arterial Pulmonar em Ratos Wistar
}

\author{
Chronic Use of Nandrolone Decanoate as Risk Factor for Pulmonary \\ Arterial Hypertension in Wistar Rats
}

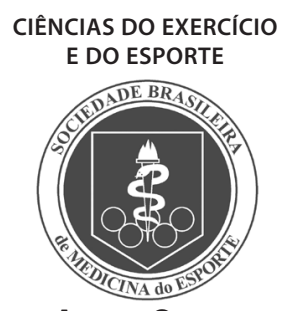

Artigo Original
Jones Bernardes Graceli ${ }^{1}$

Pablo Lúcio Gava

Helena Lima Gomes ${ }^{1}$

Leonardo Moreira Moscon²

Patrick Wander Endlich'

Washington Luiz Silva Gonçalves?

Margareth Ribeiro Moysés ${ }^{1}$

1. Departamento de Ciências

Fisiológicas, Centro de Ciências da Saúde da Universidade Federal do Espírito Santo - UFES, Vitória, ES.

2. Departamento de Farmácia do Centro Universitário de Vila Velha UV, Vila Velha, ES.

Endereço para correspondência: Departamento de Ciências

Fisiológicas, Universidade Federal do Espírito Santo.

Avenida Marechal Campos, 1.468

- Maruípe. 29042-577 - Vitória,

Espírito Santo, Brasil.

E-mail: jbgraceli@gmail.com

\section{RESUMO}

Introdução: O uso indiscriminado de esteróides anabolizantes sintéticos, análogos à testosterona, implica aumento do risco cardiovascular e hipertrofia cardíaca. Assim, o aumento da massa ventricular direita corrigido pelo peso corporal (i.é., hipertrofia ventricular direita - HVD), poderia elevar o risco para o desenvolvimento de hipertensão arterial pulmonar (HAP). Objetivos: Examinar os efeitos do tratamento em longo prazo com decanoato de nadrolona na HVD e sua relação com a HAP em ratos. Métodos: 16 ratos Wistar com três meses de idade foram aleatoriamente divididos em dois grupos: 1) controle-sham (CONT, $n=8$ ); 2) tratados com decanoato de nandrolona (DECA, $n=8)$. O tratamento consistiu na aplicação intramuscular de Deca-durabolin ${ }^{\circ} 6.0 \mathrm{mg} \mathrm{kg}^{-1}$ de peso corporal durante quatro semanas. Após tratamento, os animais foram anestesiados com hidrato de cloral $\left(4.0 \mathrm{~mL}^{\mathrm{kg}} \mathrm{kg}^{-1}\right.$, i.p.), submetidos à cateterização da artéria femoral para registro da pressão arterial media (PAM) e frequência cardíaca (FC). O coração, os rins e o fígado foram retirados, pesados e avaliados os índices de hipertrofia, os quais foram calculados pela razão da massa do órgão pelo peso corporal $\left(\mathrm{mg}^{-\mathrm{g}^{-1}}\right)$. Resultados: Os animais tratados com DECA apresentaram aumento $(p<$ $0,01)$ do peso corporal $(338 \pm 6 \mathrm{~g})$ vs. CONT $(315 \pm 5 \mathrm{~g})$. Não houve alterações da PAM, embora houvesse ( $p$ $<0,01)$ bradicardia nos animais tratados com DECA $(321 \pm 13 \mathrm{bpm})$ vs. CONT $(368 \pm 11 \mathrm{bpm})$. Verificou-se significativa $(p<0,01)$ hipertrofia dos ventrículos e rins, mas não no fígado. A correlação entre a HVD e PAM no grupo DECA apresentou coeficiente de Pearson positivo e maior $\left(r^{2}=0,4013\right)$ quando comparado com o controle $\left(r^{2}=0,0003\right)$. Conclusões: Esses dados demonstram que o uso em longo prazo de decanoato de nandrolona induz importante bradicardia e HVD, o que sugere aumento do risco para HAP.

Palavras-chave: esteróide anabolizante, pressão sanguínea, hipertrofia ventricular, hipertensão arterial pulmonar.

\section{ABSTRACT}

Introduction: The unsystematic use of anabolic steroids, synthetic analogs of testosterone, implies enhanced cardiovascular risk and cardiac hypertrophy. Thus, increased right ventricular mass corrected by the body weight (e.g.right ventricular hypertrophy -RVH) could raise the risk for development of pulmonary arterial hypertension (PAH). Objectives: to examine the effects of long-term chronic treatment with nandrolone decanoate on the RVH and its relationship with PAH in rats. Methods: 16 three-month Wistar male rats were treated with nandrolone decanoate $\left(6.0 \mathrm{mg} / \mathrm{kg}^{-1}\right.$ body weight; DECA, $\left.\mathrm{n}=8\right)$ or control vehicle (CONT, $\mathrm{n}=8$ ). The drug and vehicle were administered by a single injection in the femoral muscle once a week for 4 weeks. After the treatment, rats were anesthetized with chloral hydrate $\left(4.0 \mathrm{~mL} / \mathrm{kg}^{-1}\right.$, ip), and catheterized in the femoral artery. Twenty-four hours later, mean arterial pressure (MAP) and heart ratio were measured. The heart, kidneys and liver were removed, weighed and the rates of hypertrophy (RH) were measured, which were calculated by the ratio of the weight of the organs by the body weight (mg.g-1). Results: DECA treatment increased body weight $(338 \pm 6 \mathrm{~g} ; \mathrm{p}<0.01)$ vs. CONT $(315 \pm 5 \mathrm{~g})$. This treatment had no effect on the MAP (CONT, $110 \pm 4 \mathrm{mmHg}, \mathrm{DECA}, 113 \pm 4 \mathrm{mmHg}$ ). However, the bradycardia of animals treated with DECA ( $321 \pm 13 \mathrm{bpm}, p<0.01$ ) was significantly lower than that of CONT ( $368 \pm 11 \mathrm{bpm})$. RH increased $(p<0.01)$ the cardiac ventricles and the kidneys, but not in the liver. The correlation between the RVH and MAP in DECA showed positive and higher Pearson's coefficient $\left(r^{2}=0.4013\right)$ vs CONT $\left(r^{2}=0.0003\right)$. Conclusions: It was concluded that chronic nandrolone decanoate treatment induced bradycardia and $\mathrm{RVH}$, which suggests increased risk for PAH.

Keywords: anabolic steroids, blood pressure, ventricular hypertrophy, pulmonary arterial hypertension. 


\section{INTRODUÇÃO}

Anabolizantes são derivados sintéticos da testosterona e foram desenvolvidos com o objetivo de minimizar seus efeitos masculinizantes (androgênicos), maximizando assim os efeitos sobre a síntese proteica e o crescimento muscular (anabólico) ${ }^{(1)}$. São compostos por dois grupos: 1) derivados esterificados e 2) derivados alcalinizados. Os primeiros (fenilpropionato de nandrolona, decanoato de nandrolona, enantato de testosterona e cipionato de testosterona) são produtos para administração intramuscular e permanecem ativos por dias/semanas, enquanto os componentes do segundo grupo (oximetolona, metandrostenolona e estanozolol) devem ser utilizados diariamente, por via oral ${ }^{(2)}$.

Os principais efeitos dos esteróides anabólico-androgênicos (EAA) variam em diferentes tecidos de acordo com o ambiente celular, com o tipo de enzima ( $5 \alpha$ reductase e/ou aromatase) e com quantidade e locais dos receptores ${ }^{(3-4)}$. Particularmente, os EAA afetam o metabolismo celular através da ligação com seus receptores intracelulares e/ou com seus receptores presentes na membrana plasmática. Embora várias tentativas tenham sido feitas para diminuir o efeito androgênio dos EAA, todos os aproximadamente 60 diferentes EAA disponíveis no mercado variam em sua estrutura química e, desse modo, em seus destinos metabólicos e efeitos fisiológicos ${ }^{(5,6)}$. A natureza química hidrofóbica dos hormônios esteróides não permite que se dissolvam facilmente nos fluídos extracelulares, sendo então transportados até o tecido-alvo, via circulação sistêmica, por proteínas carregadoras específicas. Os hormônios agem nas células-alvo ligando-se a receptores específicos que podem estar na membrana, citoplasma ou no núcleo da célula. Essa ligação desencadeia alterações na conformação das proteínas receptoras (permitindo a formação de dímeros com outros complexos do mesmo tipo), de forma que estas conseguem se ligar a sequências de DNA altamente específicas, denominadas elementos de resposta ao hormônio (hormone response element - HRE), conhecidos como efeitos genômicos ${ }^{(2,5-6)}$. Por outro lado, a ligação com os receptores presentes na membrana celular plasmática ativa canais iônicos e/ou ativam segundos mensageiros que ocasionam outras respostas celulares específicas, conhecidos como efeitos não genômicos ${ }^{(8,9)}$. Entretanto, os mecanismos moleculares relacionados com os efeitos não genômicos, assim como a sua importância fisiológica, ainda não são completamente conhecidos.

Apesar de os EAA serem substâncias ilícitas para fins não terapêuticos, i.é., para ganho de força, e ainda causarem inúmeros efeitos colaterais, alguns atletas insistem em utilizá-los para se beneficiar durante as competições (doping). Isso ocorre porque quase a totalidade dos tecidos do organismo possui receptores para hormônios androgênicos e estes permanecem ativos por semanas/meses no organismo. Um exemplo disso é que os EAA estimulam a síntese de hemoglobina (proteína carreadora de oxigênio), aumentando a oferta de oxigênio nos tecidos, acrescidos do treinamento físico em altitude, consequentemente, melhora do rendimento desportivo(10).

Atletas e não atletas interessados em aumentar o desempenho físico, a força e a massa muscular vêm cada vez mais utilizando os EAA sintéticos sem indicação médica, sendo a dose usada, associada ou não ao exercício, 40 vezes maior do que o nível basal fisiológico e 20 vezes maior que o uso terapêutico convencional|(11). A terapia androgênica pode ser utilizada no tratamento da osteoporose, da anemia causada por falhas na medula óssea ou medicamentosa ${ }^{(12,13)}$, do câncer de mama avançado ${ }^{(14)}$, em garotos com estatura exagerada (13), e até mesmo em situações especiais da obesidade ${ }^{(12)}$. Há relatos de uso de esteróides anabólicos em baixas doses por via transdérmica no tratamento de doenças cardiovasculares, tendo efeitos antiaterogênicos e como agentes antianginosos ${ }^{(15)}$. No entanto, também se associam os efeitos colaterais pelo uso prolongado como o câncer de próstata, as hipertrofias cardíacas, doença coronariana e esterilidade, entre outros ${ }^{(1,11)}$.
A hipertrofia no ventrículo esquerdo (VE), após administração de anabolizantes, está bem estabelecida e descrita na literatura atual| ${ }^{(16,17) ;}$ contudo, são raros os relatos na literatura sobre a hipertrofia ventricular direita (HVD) e suas repercussões cardiopulmonares, as quais poderiam estar relacionadas com o desenvolvimento de um quadro de hipertensão arterial pulmonar (HAP). Uma condição patológica, segundo os critérios de classificação e diagnóstico da HAP(18) é primariamente definida pelo aumento da pressão arterial pulmonar média, devido à obstrução de pequenas artérias associadas a lesões plexiformes, hipertrofia da camada média ventricular direita, fibrose da íntima, lesões trombóticas, uso de drogas entre outros ${ }^{(18,19)}$. Nesse contexto, o objetivo deste estudo foi examinar os efeitos do tratamento em longo prazo (crônico) com doses suprafisiológicas de decanoato de nandrolona no desenvolvimento da HVD e sua relação com a HAP em ratos normotensos.

\section{MÉTODOS}

A investigação foi conduzida de acordo com as normas estabelecidas pelo Guide Care and Use of Laboratory Animals e os critérios adotados pelo Colégio Brasileiro de Experimentação Animal (Cobae), sendo aprovada pelo Comitê de Ética em Pesquisa Animal do Centro Universitário de Vila Velha-UV.

\section{Animais e grupos experimentais}

Foram utilizados 16 ratos machos albinos (Rattus norvergicus albinus) da linhagem Wistar adultos provenientes do Biotério do Centro Universitário de Vila Velha, ES. Os animais foram pesados e aleatoriamente separados nos seguintes grupos experimentais: 1) grupo controle-sham (CONT, $\mathrm{n}=8$ ); 2) grupo tratado com decanoato de nandrolona (DECA, $n=8$ ). Durante o estudo, os animais receberam dieta sólida (ração Nuvilab ${ }^{\circledR}$ ) e água ad libitum, sendo alojados em caixas individuais com fundo sólido, forradas com maravalha, em sala com luminosidade (12h de luz e $12 \mathrm{~h}$ de escuro) e temperatura $\left(20\right.$ a $25^{\circ} \mathrm{C}$ ) controladas.

\section{Tratamento com decanoato de nadrolona (Deca-durabolin ${ }^{\circledR}$ )}

Os animais do grupo DECA receberam injeções intramusculares (i.m.) de decanoato de nandrolona na dose de $6.0 \mathrm{mg} \mathrm{kg}^{-1}$ de peso corpóreo, diariamente por quatro semanas. Segundo Pope e Katz ${ }^{(20)}$, $5.0 \mathrm{mg} . \mathrm{kg}^{-1}$ corporal de decanoato de nandrolona equivalem à dose abusiva administrada a atletas ( $8.0 \mathrm{mg} \mathrm{kg}^{-1}$ peso corpóreo/semana) (20 a 100 vezes maior que a dose terapêutica). No presente estudo foi utilizada uma dose aproximadamente 65 vezes maior que a indicada em terapias convencionais. O grupo controle-sham (CONT) recebeu injeções i.m. de veículo (0.2 mL. $\mathrm{kg}^{-1}$ peso corpóreo de propilenoglicol), ao mesmo momento que o grupo tratado ${ }^{(20,21)}$

\section{Registro dos parâmetros cardiovasculares}

Os ratos foram anestesiados com solução -10\% de hidrato de cloral $\left(0,4 \mathrm{~mL} .100 \mathrm{~g}^{-1}\right.$ do peso corporal via intraperitoneal - i.p.); em seguida, foram realizadas incisões cirúrgicas na região inguinal dos animais para isolamento da artéria femoral, a qual foi cateterizada com uma cânula confeccionada de cateteres de polietileno. As medidas hemodinâmicas, i.é., pressão arterial média (PAM) e frequência cardíaca (FC) foram continuamente registradas pela conexão do cateter arterial femoral com os transdutores de pressão (Spectramed - Statham, P23XL, EUA), através de um sistema computadorizado para Windows ${ }^{\oplus}$ por programa para aquisição de dados biológicos (Biopac System, Inc., MP-100_/serie 94111065 - Santa Bárbara, Califórnia, EUA) possibilitando estudo com os animais acordados ${ }^{(18)}$.

\section{Índice de hipertrofia cardíaca, renal e hepática}

Todos os animais foram sacrificados por eutanásia e submetidos à laparotomia exploratória para excisão do coração e pesagem dos ventrículos direito (VD) e esquerdo (VE, incluindo o septo), fígado, rim 
esquerdo (RE) e direito (RD). Após eutanásia, os animais foram perfundidos com 50ml de solução salina (0,9\%) por meio de um cateter de polietileno (PE-50) inserido no ventrículo esquerdo. Em sequência os órgãos foram removidos cirurgicamente, decapsulados, e os ventrículos separados mecanicamente. Foram então lavados em solução salina $(0,9 \%)$ gelada $\left(4 C^{\circ}\right)$ e colocados em uma placa de Petri estéril, para aferição de seu peso úmido e peso seco. O peso seco dos órgãos foi mensurado após permanecerem na estufa a $100^{\circ} \mathrm{C}$ por $24 \mathrm{~h}$. O índice de hipertrofia dos órgãos estudados foi calculado pela equação $\left(\mathrm{mg} \cdot \mathrm{g}^{-1}\right)$ : razão da massa do órgão pelo peso corpóreo ${ }^{(16,22)}$.

\section{Análise dos dados}

Os dados foram inseridos e analisados em software estatístico (Graph-Pad-Prism4 ${ }^{\oplus}$ ), no qual se utilizaram os testes $t$ de Student para amostras independentes e análise de variâncias (ANOVA) duas vias, seguida do teste de Tukey como post hoc. A análise multivariada foi realizada através de regressão linear e coeficiente de Pearson para determinar a correlação entre HVD e PAM. Os valores foram expressos como média \pm EPM (erro padrão da média) e as diferenças estabelecidas em $p<0,05$.

\section{RESULTADOS}

Conforme representados na figura 1, os animais inicialmente não apresentaram diferenças do peso corporal entre os grupos (CONT, $298 \pm$ $10 \mathrm{~g}$ vs. DECA, $294 \pm 7 \mathrm{~g}$ ); no entanto, após o tratamento com decanoato de nandrolona por 28 dias (quatro semanas), sem exercício físico associado, houve aumento significativo $(p<0,01)$ do peso corporal quando comparado o grupo CONT (315 $\pm 5 \mathrm{~g})$ com grupo DECA (338 \pm 6g).

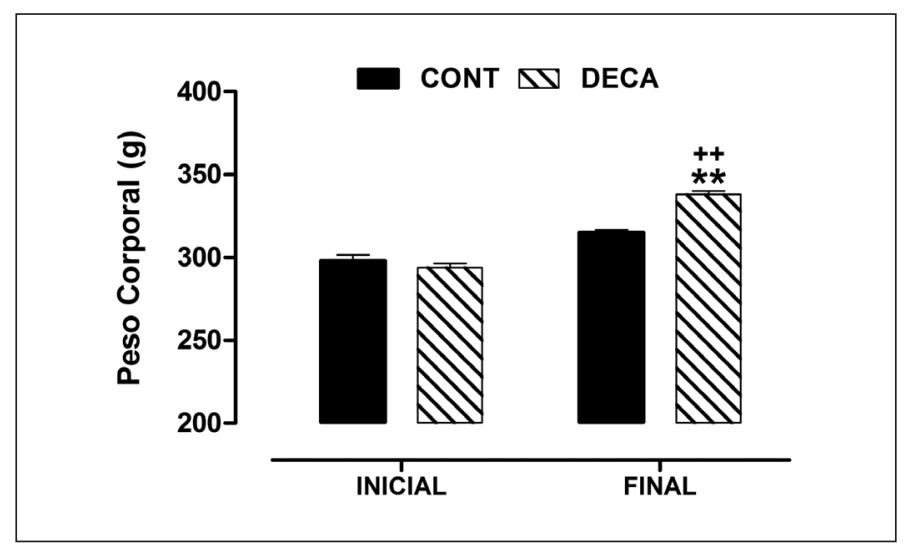

Figura 1. Peso corporal de ratos Wistar controle (CONT) e tratados com decanoato de nandrolona (DECA, 6.0 mg. $\mathrm{kg}^{-1}$ ao dia) por 28 dias. Valores expressos a média \pm EPM; Anova duas vias, e teste de Tukey com post hoc. Diferenças significativas estabelecidas em $^{* *} p<0,01$ vs. grupo $e^{++} p<0,01$ vs. tempo.
Os índices de hipertrofia dos órgãos estão apresentados na tabela 1, onde se observa aumento significativo dos pesos úmidos e secos dos ventrículos e rins direitos e esquerdos do grupo DECA quando comparados com os do grupo CONT; no entanto, para nossa surpresa, não houve alterações nos valores de massa hepática entre ambos os grupos.

Tabela 1. Determinação dos índices de hipertrofia nos órgãos internos (vísceras) de ratos Wistar controle (CONT) e após uso crônico de decanoato de nandrolona (DECA, 6,0 $\mathrm{mg} \cdot \mathrm{kg}^{-1}$, i.m.) por quatro semanas.

\begin{tabular}{l|c|c}
\hline \multicolumn{1}{c|}{ Parâmetros } & CONT & DECA \\
\hline \multicolumn{2}{|c}{ Úmido $\left(\mathbf{m g} \cdot \mathbf{g}^{-1}\right.$ ) } \\
\hline Ventrí́culo esquerdo & $1,96 \pm 0,06$ & $2,23 \pm 0,03^{* *}$ \\
\hline Ventrículo direito & $0,49 \pm 0,01$ & $0,63 \pm 0,02^{* *}$ \\
\hline Rim esquerdo & $3,5 \pm 0,09$ & $4,7 \pm 0,09^{* *}$ \\
\hline Rim direito & $3,6 \pm 0,06$ & $4,9 \pm 0,07^{* *}$ \\
\hline Fígado & $31,9 \pm 1,00$ & $35,1 \pm 1,20$ \\
\hline
\end{tabular}

\begin{tabular}{l|c|c}
\hline \multicolumn{3}{l|}{ Seco $\left(\mathbf{m g} \cdot \mathbf{g}^{-1}\right)$} \\
\hline Ventrículo esquerdo & $0,40 \pm 0,01$ & $0,44 \pm 0,01^{*}$ \\
\hline Ventrículo direito & $0,094 \pm 0,01$ & $0,120 \pm 0,01^{*}$ \\
\hline Rim esquerdo & $0,71 \pm 0,02$ & $0,94 \pm 0,03^{* *}$ \\
\hline Rim direito & $0,72 \pm 0,02$ & $0,96 \pm 0,02^{* *}$ \\
\hline Fígado & $7,9 \pm 0,2$ & $8,5 \pm 0,3$ \\
\hline
\end{tabular}

Valores expressos como média \pm EPM (erro padrão). Teste $t$ de Student para amostras independentes. Sendo *p $<0,05$ e **p $<0,01$ vs. controle.

Nos parâmetros cardiovasculares representados na figura 2, também não foram observadas alterações da pressão arterial média (PAM) em ambos os grupos (figura 2A); contudo, se verificou significativa bradicardia de repouso nos animais do grupo DECA (321 $\pm 13 \mathrm{bpm})$ quando comparados com os do grupo CONT (368 \pm 11 bpm) (figura 2B).

As correlações dos parâmetros pressóricos e dos índices de hipertrofia ventricular são apresentadas na figura 3. A PAM apresentou correlação positiva significante com a HVD $(r=0,63$; $p<0,05$, figura $3 A)$ no grupo DECA, e negativa com a HVE ( $r=-0,50$, figura $3 B$ ) no controle. A FC não se correlacionou com a HVD em ambos os grupos (figuras 3C e 3D), embora apresentasse correlação positiva com a HVE em ambos os grupos, CONT $(r=0,40)$ e DECA $(r=0,64)$; além disso, não apresentaram diferenças significativas.

\section{DISCUSSÃO}

No presente estudo, examinamos os efeitos do tratamento crônico com EAA, decanoato de nandrolona em ratos Wistar, que acarretou aumento de peso corporal com hipertrofia cardíaca e renal, no entanto, não promoveu hipertrofia hepática. Provocou ainda significativa
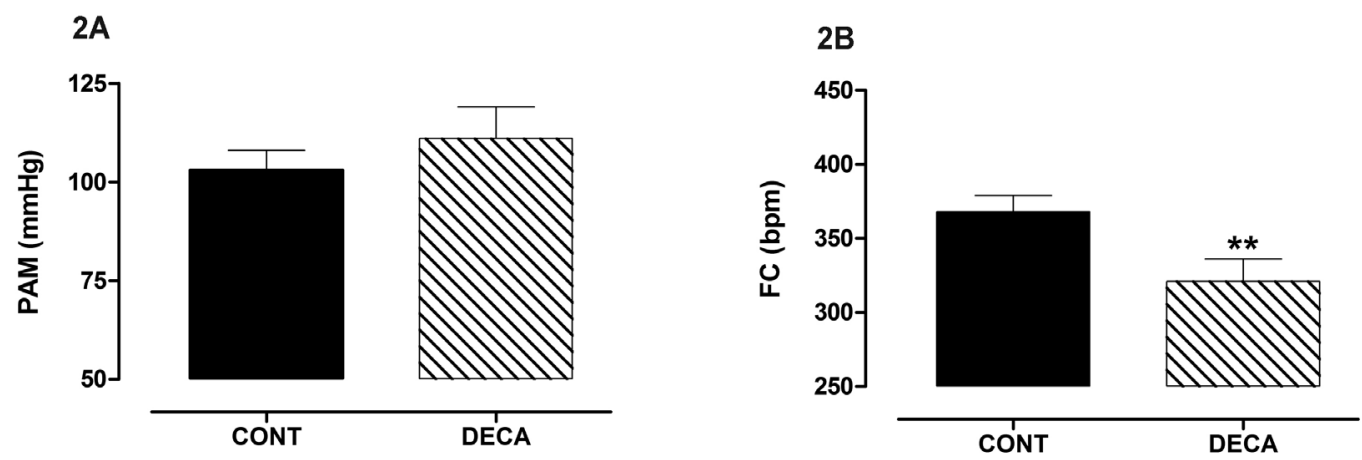

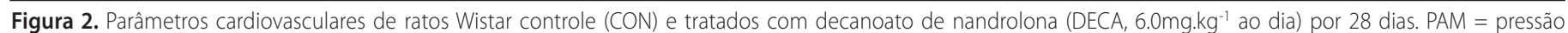

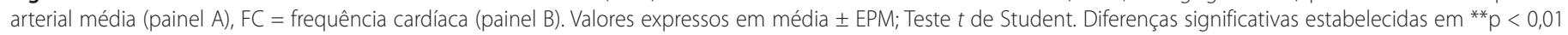
vs. grupo. 

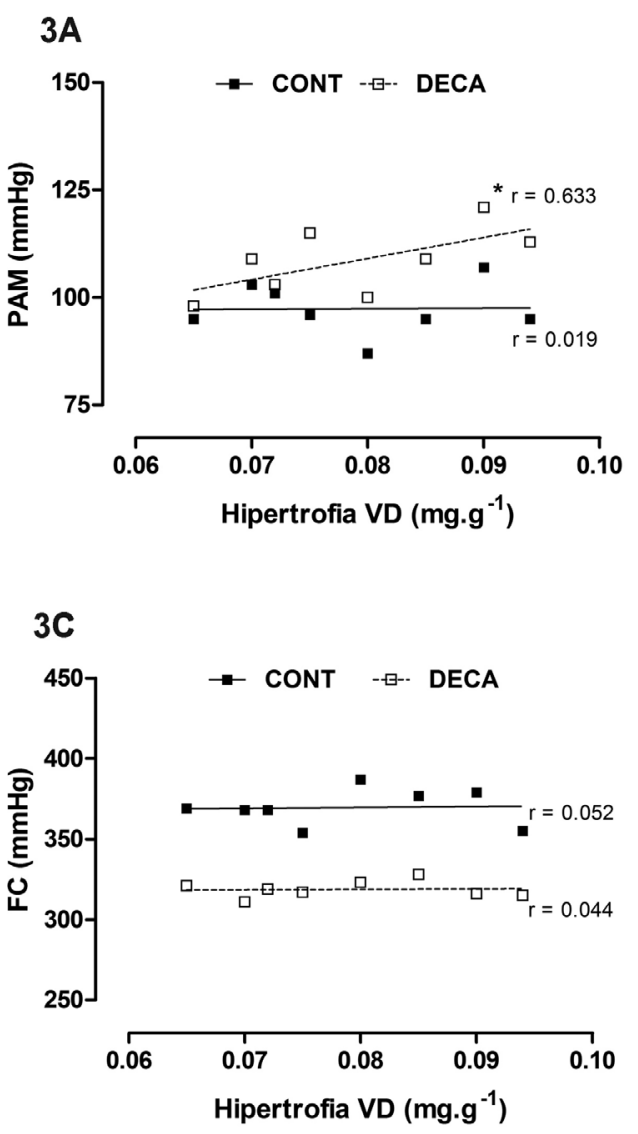
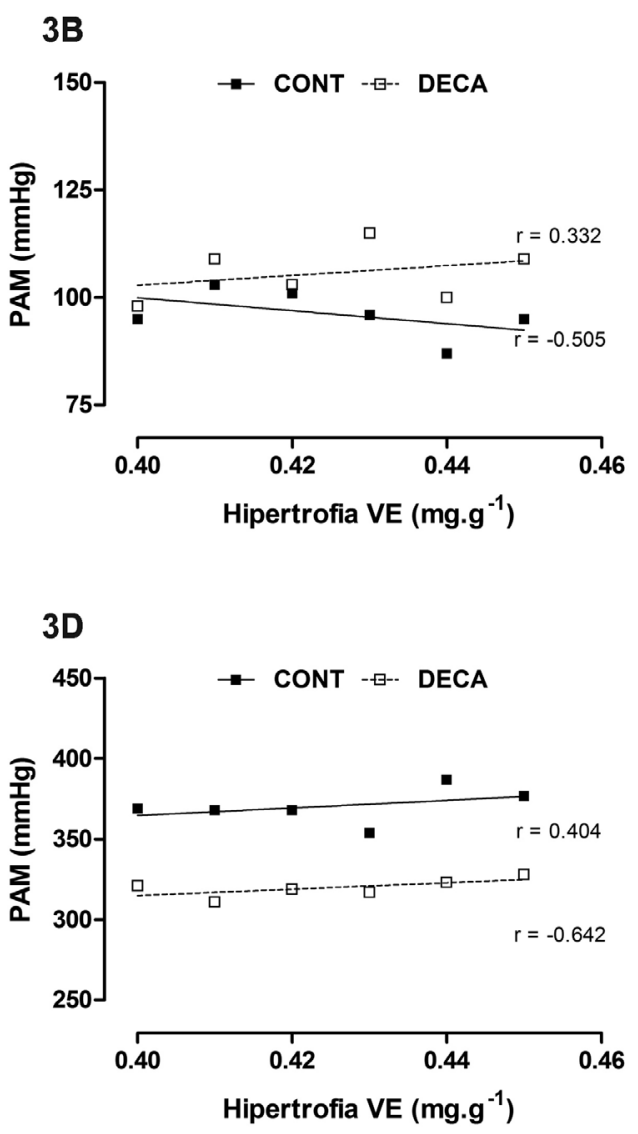

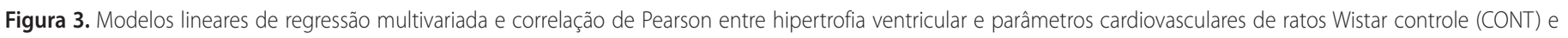

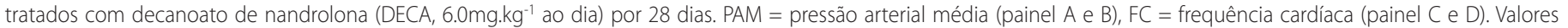
expressos em média \pm EPM. Diferenças significativas estabelecidas ${ }^{*} p<0,05$. Sendo $R^{2}=0,40$.

bradicardia com correlação significante da HVD com a PAM, o que sugeriu elevação do risco para HAP.

Corroborando nossos achados de ganho de peso nos animais tratados, outras pesquisas também mostram aumento no peso corporal e dos valores de hipertrofia do ventrículo esquerdo (HVE), após a utilização de $E A A^{(16,23)}$, além de outros efeitos cardíacos associados, como arritmias e remodelamento ventricular esquerdo ${ }^{(23)}$. A hipertrofia de VE após administração de EAA está bem descrita na literatura ${ }^{(1,11,12,17)}$, contudo, são raros os relatos sobre a hipertrofia do VD e suas repercussões hemodinâmicas e cardiopulmonares. Neste experimento encontramos significativa HVD com importante bradicardia sem alterações pressóricas significantes, sugerindo elevação da força necessária para o efluxo de sangue para os pulmões.

Estudos atuais mostram que a HAP está diretamente associada ao aumento da pós-carga do ventrículo direito e, consequentemente, a hipertrofia do mesmo(23-25). Dessa forma, a utilização do EAA decanoato de nandrolona (Deca-durabolin '), de forma crônica, pode ocasionar quadro de hipertrofia e, possivelmente, elevar os fatores de risco para a HAP em ratos normotensos. Embora a resistência pulmonar total e sistêmica não tenha sido avaliada, poderiam estar aumentadasnos animais experimentais, pois a PAM não apresentou diferença após o tratamento em longo prazo com a nandrolona. Estudos prévios de nosso grupo demonstraram que o reflexo de bradicardia (BezoldJarish) não foi modificado pela administração do EAA, decanoato de nandrolona ${ }^{(16)}$. Por outro lado, relatos na literatura sugerem que os androgênios poderiam promover o controle direto da FC pela bradicardia reflexa via barorreflexo ${ }^{(25)}$, aumentando a atividade eferente vagal cardíaca ou interferindo no drive parassimpático ${ }^{(26-28)}$. Ou, ainda, modificando a função diastólica, por mecanismos ainda não completamente elucidados, os quais poderiam influenciar de forma estrutural e/ou funcional o miocárdio, modulando seu controle intrínseco e/ou interferindo na ação dos reflexos cardiovasculares ${ }^{(28)}$. Em adição, a HVE observada poderia aumentar o débito sistólico, o que também contribuiria para a redução da FC.

Vários outros estudos têm demonstrado efeitos diretos e diferenciados dos hormônios sexuais endógenos e exógenos no sistema cardiovascular (29-31). O estrogênio, por exemplo, está diretamente associado a efeito cardioprotetor, pela redução da resistência local e periférica, pela modulação da hipertrofia de cardiomiócitos e de células de músculo liso vascular (CMLV), através do sistema renina-angiotensina cardíaco; ou ainda estimulando a formação de fatores vasodilatadores derivados do endotélio, como óxido nítrico e prostaciclinas. Por outro lado, a testosterona está mais associada ao aumento da resistência vascular periférica, causado pela estimulação da angiotensina-II, que pode ocasionar a hipertrofia de CMLV, vasoconstrição ou, ainda, estimular outros fatores vasoconstritores derivados do endotélio, como a endotelina e outros ${ }^{(30-32)}$. A hipertrofia de VE e VD observada neste estudo poderia ter sido também causada pelo aumento da síntese e deposição de colágeno na matriz extracelular das células cardíacas, num processo inicial de fibrose ventricular, que pode ser oriundo, em parte, de um estímulo positivo direto dos EAA e/ou pela ação estimulatória da angiotensinaII no músculo cardíaco e no músculo liso vascular coronariano(16,32,33). Adicionalmente, estudos recentes mostraram também que, em cultura de células cardíacas de ratos, o tratamento com testosterona e/ ou diidrotestosterona ocasionou resposta hipertrófica, em função do aumento na síntese de proteínas dos cardiomiócitos ${ }^{(33)}$. 
A hipertrofia renal após o uso em longo prazo de EAA, observada neste estudo, apresentou resultados complexos e contraditórios com os achados na literatura ${ }^{(34,35)}$. No trabalho de Blantz et al. ${ }^{(34)}$, ratos tratados por seis e por 16 semanas com deca-durabolin ${ }^{\circledR}$ apresentaram comportamentos diferentes, ou seja, o tratamento de seis semanas ocasionou aumento moderado da hipertrofia, i.é., proporcional dos segmentos tubulares e do glomérulo no néfron. No entanto, no tratamento de 16 semanas ocorreu aumento desproporcional, em que os segmentos tubulares e os glomérulos nos néfrons não apresentaram o mesmo padrão de hipertrofia, afetando a frequência de filtração glomerular, proporcional ao aumento do néfron. Nesse sentido, a hipertrofia renal também poderia ter sido causada por aumento na síntese e deposição do colágeno na matriz extracelular das células glomerulares e tubulares do néfron ${ }^{(35)}$.

Por outro lado, no fígado, surpreendentemente, o tratamento em longo prazo com Deca-durabolin ${ }^{\circledast}$ não ocasionou aumento significativo da massa do órgão, isto é, não foram encontradas diferenças entre os valores de hipertrofia hepática nos ratos controle e com doses suprafisiológicas de nandrolona. Estudos conduzidos por Sánchez-Osorio et al.(36), apresentaram resultados consistente do aumento da massa hepática, assim como elevação da deposição de colágeno hepático, após o uso prolongado de EAA. Em contrapartida, os achados de Vieira et al. ${ }^{(37)}$ mostram que suplementos orais como a creatinina não provocam alterações do trabalho das células hepáticas, i.é., hepatoxicidade, nem modificações do metabolismo de lipídeos, como as descritas para os EAAs.

Os dados obtidos nas correlações dos parâmetros cardiovasculares com os índices de hipertrofias ventriculares foram bem esclarecedores para os eventos ocorridos neste estudo. Considerando os achados de Costa et al. ${ }^{(38)}$, que demonstram que pacientes hipertensos que não apresentam queda fisiológica da pressão arterial à noite, i.é., descenso noturno, evidenciam maior prevalência de HVE. Neste estudo, apesar de os animais não serem hipertensos, verificou-se que o uso suprafisiológi-

\section{REFERÊNCIAS BIBLIOGRÁFICAS}

1. Wu FCW. Endocrine aspects of anabolic steroids. Clin Chemistry. 1997; 43(7):1289-92.

2. Snyder PJ. Androgens. In: Hardman Limbird JGLE, Goodman \& Gilman A, editors. The Pharmacological Basis of Therapeutics, 10th ed. New York: McGraw Hill, 2001;1635-48

3. Shahidi NT. A review of chemistry biological action and clinical applications of AAS. Clin Therapeuticus 2001;23(9):1355-90.

4. Bahrke MS, Yesalis CE. Abuse of AAS and related substances in sport and exercise. Curr Opin Pharmacol 2004;4:614-20.

5. Kuhn CM. Anabolic steroids. Recent Progr Horm Res. 2002;57:411-34.

6. Clarck AS, Henderson LP. Behavioral and physiological responses to anabolicandrogenic steroids. Neurosci Biobehav Rev 2003;27:413-36

7. Karbalay-Doust S, Noorafsham A. Stereological study of the effects of nandrolone decaonate on the rat prostate. Micron 2006;37:617-23.

8. O'Malley BW. Results of a search for the mechanisms of steroid receptor regulation of gene expression. Ann N Y Acad Sci 2004;1038:80-7.

9. Mendelsohn ME, Karas RH. The protective effects of estrogen on the cardiovascular system. N Engl J Med 1999;340(23):1801-11.

10. Reents S. Sport and exercise pharmacology. Champaign: Human Kinetics, 2000;344.

11. Evans NA. Current concepts in anabolic-androgenic steroids. Am J Sports Med 2004;32:534-42.

12. Corrigan B. Dehydroepiandrosterone and sport. Med J Aust. 1999;171: 206-8.

13. Conway AJ, Handelsman DJ, Lording DW, Stuckey B, Zajac JD. Use, misuse and abuse of androgens. The Endocrine Society of Australia consensus guidelines for androgen prescribing. Med J Aust 2000;172:220-4.

14. Ebeling P, Koivisto VA. Physiological importance of dehydroepiandrosterone. Lancet 1994;343:1479-81

15. English KM, Steeds RP, Jones TH, Diver MJ, Channer KS. Low-dose transdermal testosterone therapy improves angina threshold in men with chronic stable angina: a randomized, double-blind, placebocontrolled study. Circulation 2000;102:1906-11.

16. Andrade TU, Santos MCS, Busato VCW, Medeiros ARS, Abreu GR, Moysés MR, Bissoli NS. Higher physiological doses of nandrolone decanoate do not influence the Bezold-Jarish reflex control of bradycardia. Arch Med Res 2008;39(1):27-32.

17. Beutel A, Bergamaschi CT, Campos RR. Effects of chronic anabolic steroid treatment on tonic and reflex cardiovascular control in male rats. J Steroid Biochem Mol Biol 2005;93:43-48.

18. Classificação e avaliação diagnóstica da hipertensão pulmonar. J Bras Pneumol 2005,31(supl.2):S1-S8.

19. Farber HW, Loscalzo J. Mechanisms of disease. Pulmonary arterial hypertension. N Engl J Med 2004;351:1655-65.

20. Pope HG, Katz DL. Psychiatric and medical effects of anabolic-androgenic steroid use. A controlled study of 160 athletes. Arch Gen Psychiatry 1994; 51:375-82.

21. Norton GR, Trifunovic B, Woodiwiss AJ. Attenuated $\beta$-adrenoceptor-mediated cardiac contractile responses following AS administration to secondary rats. Eur J Appl Physiol 2000;81:310-6. co de EAA em longo prazo não alterou a PAM, mas produziu bradicardia com hipertrofia em ambos os ventrículos. Apesar de ser esperada uma correlação positiva entre a HVE com a PAM, isso não ocorreu em nosso modelo experimental, mesmo com o aumento do índice de HVE. Ao mesmo tempo, verificamos que a massa seca de VE apresentou apenas 10\% de hipertrofia, em relação ao controle. No entanto, na massa seca de VD observamos aumento de 27,6\% no índice de hipertrofia. Visto que os índices utilizados nas correlações da hipertrofia com a PAM foram os de massa ventricular seca, poderia justificar-se a correlação positiva significante em VD e não em VE, o que, para o VD, pequenos ajustes pressóricos podem acarretar a elevação do risco para HAP.

Diante do exposto, pode-se afirmar que o tratamento com o EAA decanoato de nandrolona em doses suprafisiológicas por quatro semanas consecutivas (28 dias) induz significante hipertrofia cardíaca e renal, sem alterações da massa hepática, causando importante bradicardia sem modificações da pressão arterial, através de mecanismos ainda desconhecidos. Por fim, conclui-se que o uso indiscriminado e abusivo do decanoato de nandrolona (Deca-durabolin ${ }^{\oplus}$ ) é fator de risco no desenvolvimento da hipertrofia cardíaca e renal, e para sobrecarga da artéria pulmonar, podendo produzir HAP em ratos normotensos.

\section{AGRADECIMENTOS}

Os autores agradecem ao Dr. F. Souza, coordenador do Laboratório de Análises Clínicas do Hospital Universitário Cassiano Antônio de Moraes-UFES, a valorosa contribuição para o estudo. Pesquisa financiada pelo Fundo de Apoio à Ciência e Tecnologia de Vitória (Facitec) e Capes e CNPq (Brasília).

Todos os autores declararam não haver qualquer potencial conflito de interesses referente a este artigo.
22. Gouvêa SA, Moysés MR, Bissoli NS, Pires JG, Cabral AM, Abreu GR. Oral administration of L-arginine decreases blood pressure and increases renal excretion of sodium and water in renovascular hypertensive rats. Braz J Med Biol Res 2003;36(7):943-9.

23. Pereira-Junior PP, Chaves EA, Costa-E-Sousa RH, Masuda MO, Carvalho ACC, Nascimento JHM. Cardiac autonomic dysfunction in rats chronically treated with anabolic steroid. Eur J Appl Physiol 2006;96:487-94.

24. Sato H, Hall CM, Griffith GW, Johnson KF, McGillicuddy JW, Bartlett RH, Cook KE. Large animal model of chronic pulmonary hypertension. ASAIO J 2008; 54(4):396-400.

25. Ward GR, Abdel-Rahman AA. Orchiectomy or androgen receptor blockade attenuates baroreflexmediated bradycardia in conscious rats. BMC Pharmacol 2006;23;6:2.

26. El-Mas MM, Afify EA, Mohy El-Din MM, Omar AG, Sharabi FM. Testosterone facilitates the baroreceptor control of reflex bradycardia: role of cardiac sympathetic and parasympathetic components. J Cardiovasc Pharmacol 2001;38:754-63.

27. El-Mas MM, Afify EA, Omar AG, Sharabi FM. Cyclosporine adversely affects baroreflexes via inhibition of testosterone modulation of cardiac vagal control. J Pharmacol Exp Ther 2002;301:346-54.

28. Kasikcioglu E, Oflaz H, Umman B, Bugra Z. Androgenic anabolic steroids also impair right ventricular function. Letters to the Editor. Elsevier Ireland Ltd. 12 February 2008.

29. Moysés MR, Barker LA, Cabral AM. Sex hormone modulation of serotonin-induced coronary vasodilation in isolated heart. Braz J Med Biol Res 2001;34(7):949-58.

30. Santos RL, Abreu GR, Bissoli NS, Moysés MR. Endothelial mediators of 17 beta-estradiol-induced coronary vasodilation in the isolated rat heart. Braz J Med Biol Res 2004;37(4):569-75.

31. Tostes RC, Nigro D, Fortes ZB, Carvalho MH. Effects of estrogen on the vascular system. Braz J Med Biol Res 2003;36(9):1143-58.

32. Freshour JR, Chase SE, Vikstrom KL. Gender differences in cardiac ACE expression are normalized in androgen-deprived male mice. Am J Physiol Heart Circ Physiol 2002;283:H1997-H2003.

33. Marsh JD, Lehmann MH, Ritchie RH, Gwathmey JK, Green GE, Schiebinger RJ. Androgen receptors mediate hypertrophy in cardiac myocytes. Circulation 1998; 98:256-61.

34. Blantz RC, Peterson OW, Blantz ER, Wilson CB. Sexual differences in glomerular ultrafiltration: effect of androgen administration in ovariectomized rats. Endocrinology 1988;122(3):767-73.

35. Dubey RK, Jackson EK. Estrogen-induced cardiorenal protection: potential cellular, biochemical, and molecular mechanisms. Am J Physiol Renal Physiol 2001;280:F365-F88.

36. Sánchez-Osorio M, Duarte-Rojo A, Martinez-Benitez B, Torre A, Uribe M. Anabolic-androgenic steroids and liver injury. Liver Int. 2007; 6:278-82.

37. Vieira RP, França RF, Carvalho CRF, Dolhnikoff M, Ribeiro W, Martins RABL. Efeitos da suplementação oral com creatina sobre o metabolismo e a morfologia hepática em ratos. Rev Bras Med Esporte 2008;14(1):38-41.

38. Costa CHRM, Batista MC, Moisés VA, Kohlmann NB, Ribeiro AB, Zanella MT. Serum insulin levels, 24-hour blood pressure profiles, and left ventricular mass in non-obese hypertensive patients. Hypertension 1995;26(part 2):1085-8. 